\title{
QeiOVID
}

\section{Milad Shirvaliloo}

1 Tabriz University of Medical Sciences

Potential competing interests: The author(s) declared that no potential competing interests exist.

\section{Definitions}

Qeios

Defined by Alberto Bedogni et al.

A portmanteau of "Qeios" and "COVID", QeiOVID (kay-oh-vid) is a collective term that refers to every kind of research concerned with the Coronavirus Disease 2019 (COVID-19) published first on Qeios. 